\title{
Infecção por Cytauxzoon spp. em felinos domésticos
}

\author{
[Infection by Cytauxzoon spp. in domestic felines]
}

\section{"Revisão/Review"}

\section{Taiã Mairon Peixoto Ribeiro ${ }^{1 *}$, Helcileia Dias Santos², Thássia Silva Reis ${ }^{2}$, Sebastiana Adriana Pereira Sousa ${ }^{1}$, Maria Eduarda Chiaradia Furquim ${ }^{\mathbf{3}}$, Marcos Rogério André3 Valéria de Sá Jayme ${ }^{1}$}

\author{
${ }^{1}$ Departamento de Medicina Veterinária Preventiva, Escola de Veterinária e Zootecnia, Universidade Federal de Goiás, \\ Goiânia-GO, Brasil. \\ ${ }^{2}$ Curso de Medicina Veterinária, Escola de Medicina Veterinária e Zootecnia, Universidade Federal do Tocantins, \\ Araguaína-TO, Brasil. \\ ${ }^{3}$ Laboratório de Imunoparasitologia, Departamento de Patologia Veterinária, Faculdade de Ciências Agrárias e \\ Veterinárias, Universidade Estadual Paulista “Julio de Mesquita Filho", Jaboticabal-SP, Brasil. \\ *Autor para correspondência/Corresponding author. E-mail: ribeiro.vet@uft.edu.br
}

\section{Resumo}

Cytauxzoon spp. são piroplasmas hemoparasitos que parasitam espécimes da família Felidae. A cytauxzoonose é uma enfermidade caracterizada principalmente por sinais clínicos inespecíficos e súbitos. Tem como reservatórios os felídeos silvestres, no entanto já existem relatos de parasitemia crônica em felinos domésticos. A transmissão ocorre principalmente por vetores como carrapatos na América do Norte; já na América do Sul, Europa e Ásia os vetores são ainda desconhecidos. A patogenia decorre da fase de esquizogonia do parasito em macrófagos que causa obstrução de vasos sanguíneos. O tratamento é baseado nos cuidados de suporte e a combinação terapêutica de atovaquona associada à azitromicina tem demonstrando bons resultados. A profilaxia consiste na restrição do acesso de gatos domésticos em áreas arborizadas que possuam a presença de carrapatos e no uso de repelentes-acaricidas. Com esta revisão de literatura, objetivou-se analisar os aspectos epidemiológicos, clínicos e patológicos que permitam subsidiar o médico veterinário atuar com rapidez em casos suspeitos de cytauxzoonose.

Palavras-chave: cytauxzoonose; gatos; Felidae.

\begin{abstract}
Cytauxzoon spp. are piroplasms, hemoparasites parasitizing specimens of the Felidae family. Cytauxzoonosis is a disease characterized mainly by nonspecific and sudden clinical signs. It has as reservoirs the wild felids, however there are already reports of chronic parasitemia in domestic felines. Transmission occurs mainly by vectors such as ticks in North America; but in South America, Europe, and Asia, the vectors are still unknown. The pathogenesis stems from the schizogony stage of the parasite in macrophages that causes obstruction of blood vessels. Treatment is based on supportive care and the combination therapy of atovaquone associated with azithromycin has shown good results. Prophylaxis consists in restricting the access of domestic cats to wooded areas with the presence of ticks and the use of repellents and acaricides. With this literature review, the objective was to analyze the epidemiological, clinical, and pathological aspects that allow the veterinarian to act quickly in cases suspected of cytauxzoonosis.
\end{abstract}

Keywords: cytauxzoonosis; cats; Felidae.

\section{Introdução}

O gato doméstico (Felis silvestris catus) distribuídas mundialmente devido a sua associação constitui uma das espécies de mamíferos mais com a espécie humana, estando presente em todos 
os continentes (Ottoni et al., 2017). De acordo com Driscoll et al. (2009) a população mundial de felinos domésticos é estimada em torno de 600 milhões de espécimes e, segundo Brasil (2015), a população felina brasileira é estimada em 22,1 milhões, sendo utilizados pela sociedade humana pincipalmente como animal de estimação e como agente no controle de roedores.

Os gatos domésticos podem adquirir patógenos transmitidas por vetores. Dentre eles destaca-se o hemoparasito Cytauxzoon felis, o qual está relacionado ao acometimento de febre, depressão, apatia, letargia e icterícia, e a uma alta taxa de letalidade em gatos (Rizzi et al., 2015). A cytauxzoonose é considerada uma enfermidade descrita há 40 anos no continente americano, em especial nos Estados Unidos da América (USA) (Legroux et al., 2017). Adicionalmente, a enfermidade vem se expandindo no continente europeu desde o início do século XXI, com recentes relatos clínicos em felinos domésticos nos países deste continente (Carli et al., 2012; Lloret et al., 2015; Alho et al., 2016). Já no Brasil, o agente foi inicialmente detectado em felinos selvagens (Peixoto et al., 2008; André et al., 2009) e vem sendo detectado, desde então, em felinos domésticos (André et al., 2015; André et al., 2017).

Devido a relevância em medicina felina e a alta taxa de letalidade apresentada por significativa parcela dos animais infectados, objetivou-se a realização de revisão de literatura acerca dos aspectos etiológicos, epidemiológicos, clínicos e laboratoriais desta hemoparasitose.

\section{Agente etiológico}

O gênero Cytauxzoon spp. pertence à ordem Piroplasmida e família Theileriidae (CriadoFornelio et al., 2003; Schreeg et al., 2016). Após o repasto sanguíneo de um carrapato infectado, os esporozoítos de Cytauxzoon felis adentram diretamente em monócitos e macrófagos de um felídeo, onde sofrem esquizogonia e dão origem à formação de esquizontes maduros que rompem os macrófagos infectados e liberam merozoítos (Tarigo et al., 2013; Reichard et al., 2010; O'Donoghue, 2017). Os merozoítos, por sua vez, entram em eritrócitos e se replicam induzindo à morte celular, resultando em liberação de novos merozoítos na circulação sanguínea, que por sua vez adentram em outros eritrócitos (Tarigo et al., 2013). Alguns merozoítos podem vir a se diferenciar em gametócitos no intestino do vetor após ingestão por carrapatos, onde já no organismo do invertebrado se modificam para corpos raiados que se fundem para gerar um zigoto diploide no intestino do vetor (Wang et al., 2017). Os zigotos diploides dão origem aos cinetos haploides que migram para glândulas salivares onde se modificam para esporozoítos (Tarigo et al., 2013; Wang et al., 2017).

As espécies de carrapatos Dermacentor variabilis e Amblyomma americanum são até o momento os únicos vetores confirmados de C.felis, sendo que a transmissão ocorre por meio de ninfas e adultos de carrapatos infectados (transestadial), e não há transmissão transovariana (Blouin et al., 1984; Reichard et al., 2009; Reichard et al., 2010; Schreeg et al., 2016). Larvas infectadas, após sofrerem ecdise e se transformarem em ninfas permanecem infectivas, demostrando a transmissão transestadial de $C$. felis (Allen et al., 2019). É necessário um tempo mínimo entre $>36 \mathrm{~h}$ a $\leq 48 \mathrm{~h}$ de contato de espécimes adultos de $A$. americanum com o hospedeiro para que haja transmissão de C. felis (Thomas et al., 2018). Nos Estados Unidos da América (EUA), Zieman et al. (2017) constataram, utilizando-se a técnica de reação em cadeia de polimerase convencional (PCR), positividade para C. felis em $A$. americanum e $D$. variabilis coletados diretamente na vegetação local da Região Sul de Illinois (EUA).

\section{Histórico da doença e epidemiologia}

Casos de cytauxzoonose em felinos foram diagnosticados pela primeira vez entre os anos de 1973-1975 em quatro gatos domésticos do Missouri (USA) com semelhantes perfis epidemiológicos, clínicos e patológicos, evidenciação no exame microscópico de esquizontes de Cytauxzoon spp. em tecidos viscerais e presença de inclusões intraeritrocíticas (piroplasmas) nas hemácias dos animais acometidos (Wagner, 1976). Em linces-vermelhos (Lynx rufus), considerados reservatórios de C. felis na América do Norte, o parasita apresenta possivelmente uma esquizonia mais limitada, embora existam casos relatados de óbito por cytauxzoonose nesta espécie animal (Blouin et al., 1987; Nietfeld e Pollock, 2002). Embora os espécimes de Lynx rufus possam permanecer cronicamente infectados, eles não são imunes a reinfecções com amostras diferentes de C. felis (Zieman et al., 2018).

Embora se acreditasse que sua presença era restrita ao continente americano, casos positivos nos continentes Europeu e Asiático têm sido 
relatados, como em Portugal (gato doméstico, Felis silvestris catus), França (Felis silvestris catus), Itália (Felis silvestris catus e gato bravo europeu, Felis silvestris silvestris), Romênia (Lince Euroasiático, Lynx lynx e Felis silvestris silvestris), Espanha (lince Ibérico, Lynx pardinus e Felis silvestris catus), Suíça (Felis silvestris catus), Alemanha (tigre, Panthera tigris), Bósnia e Herzegovina (Felis silvestris silvestris), Zimbábue (leão, Panthera leo), Irã (Felis silvestris catus e Felis silvestris sp.), Mongólia (gato de pallas, Otocolobus manul), China (Felis silvestris catus) e em outras espécies que não pertencem à família Felidae, como no Japão (urso marrom de Hokkaido, Ursus arctos yesoensis) e África do Sul
(Suricata suricatta) (Jakob e Wesemeier, 1996; Ketz-Riley et al., 2003; Reichard et al., 2005; Joyner et al., 2007; Criado-Fornelio et al., 2009; Meli et al., 2009; Jinnai et al., 2010; Carli et al., 2012; Carli et al., 2014; Kelly et al., 2014; LeClaire et al., 2014; Rassouli et al., 2015; Zaeemi et al., 2015; Alho et al., 2016; Gallusová et al., 2016; Veronesi et al., 2016; Díaz-Regañón et al., 2017; Legroux et al., 2017; Hodžić et al., 2018; Nentwig et al., 2018; Zou et al., 2019).

No Brasil, foi constatada positividade para Cytauxzoon sp. em diversas espécies, sendo sugerido por Furtado et al. (2017) que a onçapintada (Panthera onca) pode ser um reservatório (Tabela 1).

Tabela 1. Felídeos infectados por Cytauxzoon spp. no Brasil entre 2007-2018.

\begin{tabular}{|c|c|c|c|c|}
\hline $\begin{array}{l}\text { Nome científico/ } \\
\text { Nome Popular }\end{array}$ & Teste Diagnóstico & $\begin{array}{c}\text { Cidade-Estado ou } \\
\text { Região }\end{array}$ & $\begin{array}{c}\mathrm{N}^{0} \text { de Positivos/ } \\
\mathrm{N}^{0} \text { de Animais } \\
\text { Testados }\end{array}$ & Referência \\
\hline \multirow{3}{*}{$\begin{array}{l}\text { Gato doméstico (Felis } \\
\text { silvestris catus) }\end{array}$} & \multirow{3}{*}{$\begin{array}{l}\text { Esfregaço } \\
\text { sanguíneo }\end{array}$} & $\begin{array}{c}\text { Rio de Janeiro-RJ / } \\
2002\end{array}$ & $6 / 38$ & \multirow{3}{*}{$\begin{array}{c}\text { Mendes-de-Almeida et } \\
\text { al. (2007) }\end{array}$} \\
\hline & & $\begin{array}{l}\text { Rio de Janeiro-RJ / } \\
2003\end{array}$ & $17 / 47$ & \\
\hline & & $\begin{array}{l}\text { Rio de Janeiro-RJ / } \\
2004\end{array}$ & $24 / 33$ & \\
\hline $\begin{array}{l}\text { Gato doméstico (Felis } \\
\text { silvestris catus) }\end{array}$ & PCR & Areal-RJ & $1 / 1$ & Maia et al. (2013) \\
\hline $\begin{array}{l}\text { Gato doméstico (Felis } \\
\text { silvestris catus) }\end{array}$ & PCR & Campo Grande-MS & $1 / 151$ & André et al. (2015) \\
\hline $\begin{array}{l}\text { Gato doméstico (Felis } \\
\text { silvestris catus) }\end{array}$ & PCR & Mossoró-RN & $2 / 3$ & André et al. (2017) \\
\hline $\begin{array}{c}\text { Jaguatirica (Leopardus } \\
\text { pardalis) }\end{array}$ & PCR & Brasília-DF & $2 / 3$ & André et al. (2009) \\
\hline $\begin{array}{c}\text { Jaguatirica (Leopardus } \\
\text { pardalis) }\end{array}$ & PCR & Jundiaí-SP & $4 / 24$ & André et al. (2009) \\
\hline $\begin{array}{c}\text { Jaguatirica (Leopardus } \\
\text { pardalis) }\end{array}$ & PCR & São Paulo-SP & $1 / 5$ & Filoni et al. (2012) \\
\hline $\begin{array}{c}\text { Jaguatirica (Leopardus } \\
\text { pardalis) }\end{array}$ & PCR & $\begin{array}{c}\text { Santarém- } \\
\text { PA/Rurópolis-PA }\end{array}$ & $1 / 1$ & Soares et al. (2017) \\
\hline $\begin{array}{c}\text { Jaguatirica (Leopardus } \\
\text { pardalis) }\end{array}$ & PCR & Corumbá-MS & $4 / 7$ & De Sousa et al. (2018) \\
\hline Leão (Panthera leo) & $\begin{array}{l}\text { Histopatológico e } \\
\text { Microscopia } \\
\text { Eletrônica }\end{array}$ & Volta Redonda-RJ & $1 / 1$ & Peixoto et al. (2007) \\
\hline Onça (Panthera onca) & PCR & Brasília-DF & $1 / 1$ & André et al. (2009) \\
\hline & & Pantanal & $44 / 44$ & \\
\hline Onça (Panthera onca) & PCR & Cerrado & $22 / 22$ & Furtado et al. (2017) \\
\hline & & Amazônia & $3 / 4$ & \\
\hline $\begin{array}{c}\text { Onça-parda (Puma } \\
\text { concolor })\end{array}$ & PCR & Brasília-DF & $2 / 3$ & André et al. (2009) \\
\hline $\begin{array}{c}\text { Onça-parda (Puma } \\
\text { concolor) }\end{array}$ & PCR & Campo Grande-MS & $1 / 1$ & Antunes et al. (2018) \\
\hline
\end{tabular}


No Brasil, assim como na Europa e Ásia, ainda permanecem desconhecidos os vetores de Cytauxzoon sp. (Peixoto et al., 2007; André et al. 2015; Alvarado-Rybak et al., 2016; André et al., 2017).

Acreditava-se que a letalidade em gatos domésticos seria de $100 \%$, no entanto tem-se observado a presença de portadores crônicos da enfermidade com longo período de hemoparasitemia (15 meses ou mais) (Meinkoth et al., 2000; Rizzi et al., 2015).

Alguns autores sugerem que $C$. felis seja mais virulento do que Cytauxzoon sp. amostra europeia, pois não há relatos de esquizogonia em casos clínicos na Europa (Nentwig et al., 2018). Adicionalmente, Cytauxzoon sp. amostra europeia tem maior proximidade genética ao Cytauxzoon sp. encontrado no Velho Mundo (Carli et al., 2012; Lloret et al., 2015; Legroux et al., 2017).

Existe uma proximidade genética maior entre as espécies de Cytauxzoon spp. encontradas no Brasil e na América do Norte do que entre a Europa e América do Norte (Carli et al., 2012; André et al., 2015; Furtado et al., 2017; Soares et al., 2017). A patogenicidade dos genótipos de Cytauxzoon sp. no Brasil permanece desconhecida (Maia et al., 2013; André et al., 2015).

Pesquisas já foram realizadas na tentativa de verificar a transmissão vertical por Cytauxzoon spp., todavia ainda não foi observada a transmissão transplacentária nestes estudos (Weisman et al., 2007; Lewis et al., 2012). Não foi verificada a transmissão pela ingestão de carrapatos infectados com Cytauxzoon felis por gatos domésticos em estudo conduzido por Thomas et al. (2018). Estudos sugerem que não há associação com raça, idade, presença ou ausência de retrovírus ou gênero na infecção por Cytauxzoon spp. nos animais, mas sim, em relação com o acesso ao ambiente externo ao domicílio, próximo ou dentro de áreas com pastagens, com arborização e presença de reservatórios silvestres (Meinkoth et al., 2000; Reichard et al., 2008; Carli et al., 2012; Raghavan et al., 2014).

\section{Patogenia}

A patogênese da cytauxzoonose está diretamente relacionada à fase de esquizogonia, pois os esporozoítos, ao infectarem células mononucleares associadas ao endotélio vascular sofrem replicação desenvolvendo grandes estruturas conhecidas como esquizontes, e assim obstruem vasos sanguíneos em vários órgãos. De fato, a presença de esquizontes em macrófagos mostra-se um achado laboratorial patognômico da doença (Meinkoth e Kocan, 2005; Shock et al., 2011; Lloret et al., 2015). Schreeg et al. (2016) sugeriram que Cytauxzoon sp. induza a macrófagos e monócitos infectados a não morrer por apoptose, enquanto os esquizontes se replicam e formam merozoítos infectantes. Podem ocorrer diferentes expressões de genes durante a fase de esquizogonia, o que poderia resultar em diferentes graus de patogenicidades entre as amostras de $C$. felis (Pollard et al., 2017).

Os imprints de órgãos e lâminas histopatológicas (derivados de tecidos coletados por biópsia ou necrópsia), especialmente de pulmão e baço, podem evidenciar que macrófagos quando infectados com Cytauxzoon spp. tornam-se aumentados, com um núcleolo grande e proeminente (Cohn, 2011). Inicialmente, o esquizonte é azul pequeno, lobulado e mal definido no espaço intracitoplasmático e, à medida que vai amadurecendo, tornam-se pequenos (Figura 1), com áreas nucleares roxas dentro do esquizonte (Meinkoth e Kocan, 2005).

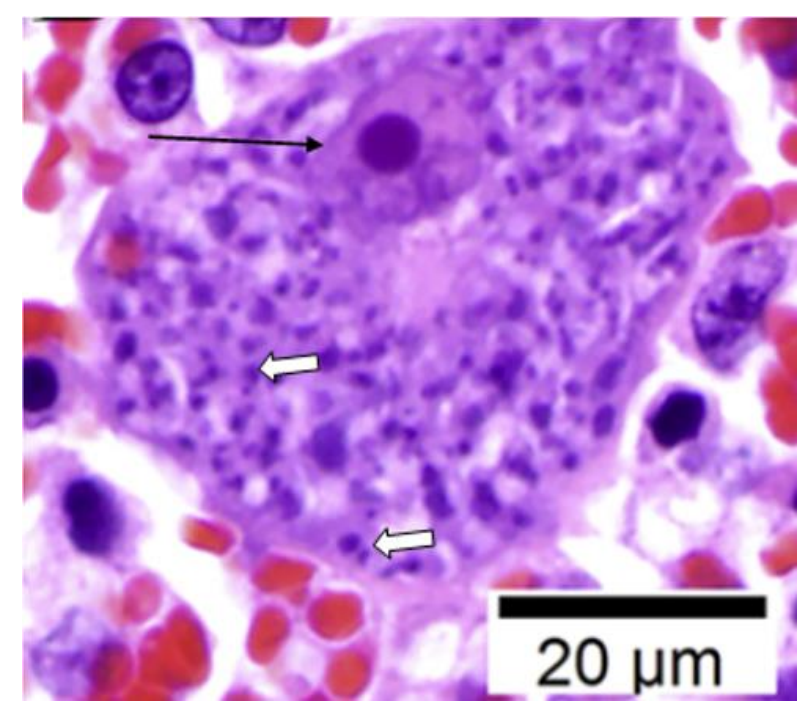

Figura 1. Macrófago contendo esquizontes de C. felis, onde observam-se merozoítos (setas brancas) e nucléolo proeminente (setas pretas). Fonte: Adaptado de Khana et al. (2018).

A oclusão vascular e vasculite durante a fase de esquizogonia são reconhecidas como principais mecanismos patofisiológicos da cytauxzoonose, devido a isquemia tecidual nos locais afetados, possibilitando o paciente felino vir à óbito antes da detecção da eritroparasitemia pelos métodos parasitológicos (Meinkoth e Kocan, 2005).

Estudos demonstraram que gatos que sofreram esquizogonia e sobreviveram podem se 
tornar imunes a uma segunda infecção (Cohn e Birkenheuer, 2012). No entanto, existem relatos de que alguns animais que tiveram cytauxzoonose podem vir a ter uma recidiva da mesma doença após ter se recuperado da infecção aguda (Cohn, 2011).

O aumento do número de eritrócitos contendo piroplasmas pode ser verificado após o início de tratamento de linfoma intestinal, sugerindo que a imunossupressão pode exacerbar a parasitemia (Rizzi et al., 2015).

A transfusão de sangue de um gato cronicamente infectado para um gato indene pode resultar em doença ou não, existindo sempre um risco de que a parasitemia pode preceder o aparecimento dos sinais clínicos (Wardrop et al., 2016). Enquanto a inoculação de tecidos contendo esquizontes é associada a quadros graves da doença, a inoculação de merozoítos intraeritrocíticos resulta apenas em leve parasitemia, mas não resulta em esquizogonia (Cohn e Birkenheuer, 2012). Por outro lado, outros pesquisadores acreditam que a eritroparasitemia não confere imunidade ao hospedeiro felino, que pode ir ao óbito quando desafiado com a inoculação de tecidos com esquizontes (Meinkoth et al., 2000; Meinkoth e Kocan, 2005).

São observados altos títulos de anticorpos perto do pico de parasitemia e acredita-se que esquizontes de $C$. felis induzam imunidade competente para debelar infecção esquizônica de C. felis (Cowell et al., 1988). No entanto, piroplasmas em hemácias não são detectáveis em esfregaços sanguíneos na fase aguda (esquizogonia) da enfermidade, a qual precede a eritroparasitemia (Wang et al., 2017).

\section{Sinais clínicos}

A febre acentuada é o achado clínico mais comum, contudo em gatos moribundos e em estágio final a temperatura poderá cair, tendo geralmente uma apresentação clínica em forma de síndrome febril aguda (febre entre $39,4{ }^{\circ} \mathrm{C}$ 41, $6^{\circ} \mathrm{C}$ ) (Wagner et al., 1980; Cohn, 2011; Lloret et al., 2015). Podem ser observados ao exame físico e anamenese sinais não específicos, tais como letargia, desidratação, icterícia, anorexia, dispneia, taquipneia, vocalização, mucosas pálidas ou ictéricas, pneumonia e edema pulmonar (Wagner et al., 1980; Meinkoth, 2000; Meinkoth e Kocan, 2005; Snider et al., 2010; Cohn e Birkenheuer, 2012; Clarke e Rissi, 2015).
Tais alterações podem evoluir para a cronicidade com sinais neurológicos como panuveíte hemorrágica, ataxia, nistagmo, perda de consciência, convulsões, urina com coloração amarelo-escuro, retardamento no tempo de preenchimento capilar, hipotermia, hiperestesia na palpação muscular, esplenomegalia, hepatomegalia, linfadenomegalia, estado apático e coma (Wagner et al., 1980; Meinkoth, 2000; Meinkoth e Kocan, 2005; Snider et al., 2010; Cohn, 2011; Cohn e Birkenheuer, 2012; Clarke e Rissi, 2015; Meekins e Cino-Ozuna, 2018).

O período médio de incubação é de 10 a 15 dias, variando de cinco dias a até 21 dias; contudo, muitos gatos morrem antes mesmo de aparecerem os primeiros sinais clínicos (Rizzi et al., 2015; Wang et al., 2017; Allen et al., 2019). Após a detecção dos primeiros sinais clínicos os pacientes felinos podem vir à óbito em 24 horas ou em até sete dias (Nagamori et al., 2016). Os achados clínicos e o histórico, especialmente a síndrome febril, não são patognômicos para cytauxzoonose felina, por isso diversas doenças devem ser levadas em conta no momento do diagnóstico.

No Brasil, casos confirmados de cytauxzoonose foram confirmados somente em Panthera leo no estado do Rio de Janeiro, por meio de diagnóstico histopatológico (Peixoto et al., 2007). Em gatos domésticos, a despeito da detecção do agente por esfregaços sanguíneos (piroplasmas em eritrócitos) (André et al., 2017) e métodos moleculares (André et al., 2015, André et al., 2017), casos confirmados de cytauxzoonose por meio da observação de esquizontes em macrófagos teciduais ainda são inexistentes.

\section{Diagnóstico laboratorial e achados patológicos}

O clínico veterinário poderá utilizar vários recursos de diagnóstico, especialmente, os exames de patologia clínica, histopatologia, exames macroscópicos e diagnóstico por imagem. A radiografia ou ultrassonografia abdominal podem revelar hepatomegalia, esplenomegalia e padrões de pneumonia intersticial, a qual pode ser definida como espessamento das paredes septais alveolares por células inflamatórias ou edema alveolar (Snider et al., 2010; Lloret et al., 2015).

Os sinais encontrados na patologia clínica incluem anemia não regenerativa, pancitopenia, leucopenia, trombocitopenia, hiperbilirrubinemia, bilirrubinúria, azotemia pré-renal, hiperglicemia, elevação das enzimas hepáticas e aumento no tempo de coagulação, especialmente protrombina e 
baixa atividade da proteína C (Cohn, 2011; Cohn e Birkenheuer, 2012; Lloret et al., 2015).

A hiperbilirrubinemia é derivada da hemólise e infiltração intra-hepática de macrófagos contendo esquizontes (Cohn, 2011). Os aspirados de medula óssea, linfonodos, baço e fígado também podem ser usados, evidenciando esquizontes em macrófagos (Meinkoth e Kocan, 2005; Grindem et al., 2008).

O exame de esfregaço sanguíneo para observação de merozoítos intra-eritrocitários é bastante utilizado, mas a limitação deste método é que os níveis de piroplasmas circulantes são baixos, podendo estar ausentes no período inicial da infecção, haja vista que os sinais clínicos causados pela esquizogonia podem preceder a detecção de Cytauxzoon spp. nas hemácias (Carli et al., 2014; Lloret et al., 2015). Porém, uma vez que com o prolongamento da doença, é esperado um aumento neste nível de piroplasmas intraeritrocíticos, devendo ser realizados esfregaços diários para verificar a presença do agente etiológico ou patógeno e evolução da doença (Carli et al., 2014).

A qualidade do diagnóstico parasitológico de Cytauxzoon sp. também depende da qualidade do esfregaço sanguíneo, a fim de evitar a formação de artefatos na técnica. As células sanguíneas devem estar dispostas homogeneamente sobre as lâminas a fim de evitar a sobreposição de hemácias. É fundamental proceder a secagem das lâminas antes da coloração para evitar acúmulo de água na lâmina, que resulta em estruturas intra-eritrocíticas que podem ser confundidas com piroplasmídeos (Meinkoth e Kocan, 2005). Os corantes devem ser trocados periodicamente para evitar a formação de precipitados nas lâminas, que se apresentam como manchas entre as células ou sobre as células, sendo diferenciadas de parasitos pela ausência de citoplasma e presença de contornos irregulares (Meinkoth e Kocan, 2005).

Corpúsculos de Howell-Jolly, que são remanescentes de material nuclear intraeritrocíticos devem ser diferenciados de piroplasmas de Cytauxzoon spp. Tais estruturas se apresentam geralmente como pontos únicos, redondos, escuros, com tamanho variável e não possuem citoplasma (Meinkoth e Kocan, 2005; Wang et al., 2017).

Piroplasmas intra-eritrocíticos de Cytauxzoon sp. devem ser diferenciados de outros hemoparasitas transmitidos por vetores, tais como hemoplasmas (micoplasmas hemotróficos) e outros piroplasmídeos (Theileria spp. e Babesia spp.) (Wang et al., 2017). Dentre as diferenças entre Mycoplasma spp. e Cytauxzoon spp. destacase que os micoplasmas são menores, se posicionam na área epicelular e geralmente formam pontos, enquanto que os Cytauxzoon spp. apresentam formato de anel e citoplasma visível (Figura 2) (Meinkoth e Kocan, 2005; Cohn, 2011). No Brasil, merozoítos de Babesia spp. e Theileria spp. devem ser distinguidos daqueles de Cytauxzoon spp., haja vista a ocorrência dos três gêneros de piroplasmídeos em felinos domésticos em nosso território (André et al., 2014; André et al., 2015; Malheiros et al., 2016; André et al., 2017). A fim de um diagnóstico etiológico mais acurado, preconiza-se a utilização de testes moleculares seguida de sequenciamento e posicionamento filogenético (André et al., 2017).

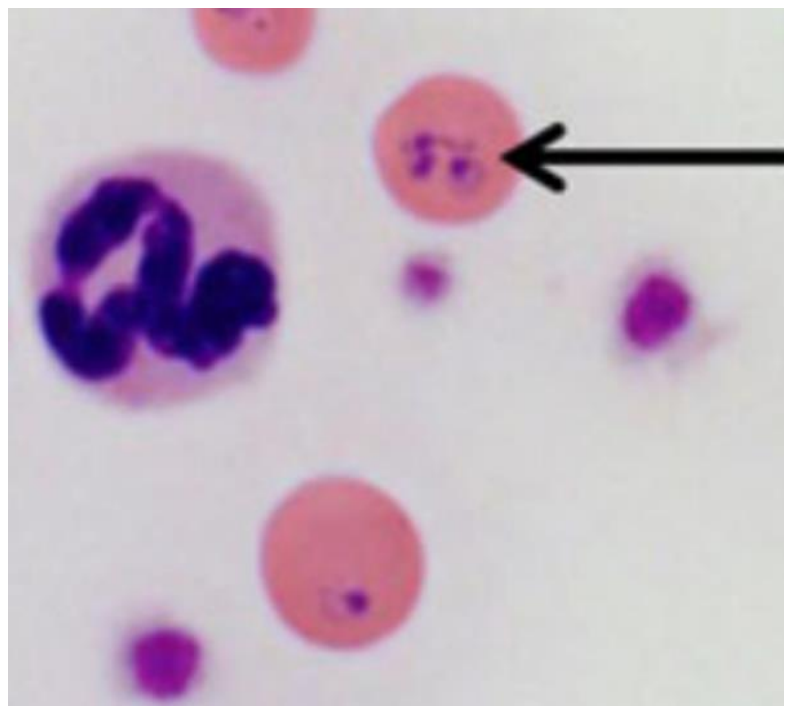

Figura 2. Hemácias com piroplasmas característicos de Cytauxzoon sp. em forma de anel (seta preta) em esfregaço sanguíneo de paciente felino. Fonte: Adaptado de Nentwig et al. (2018).

Hemorragias petequiais e equimoses podem ocorrer em vários tecidos, especialmente na superfície pulmonar, além de infiltração neutrofílica e macrofágica em paredes de septos alveolares e no interstício peribronquial (Snider et al., 2010; Aschenbroich et al., 2012). Durante a necropsia podem ser verificadas icterícia difusa, esplenomegalia, baço com coloração vermelha escura, fígado amarelo acastanhado e pulmões com focos hemorrágicos em sua superfície (Aschenbroich et al., 2012).

As alterações histológicas cerebrais incluem macrófagos contendo esquizontes em arteríolas e vênulas parenquimatosas, vasculite e trombos de fibrina, oclusão de capilares na substância cinzenta 
e branca, com presença de linfócitos, astrocitose, microgliose, astrogliose, microhemorragias múltiplas, neurônios necróticos e potencial indução de apoptose em células do sistema nervoso (Clarke e Rissi, 2015; Clarke et al., 2017).

Existe uma dificuldade técnica em se produzir antígenos purificados para o diagnóstico imunológico, por isso não existe kit disponível comercialmente (Shindel et al., 1978; Wagner et al., 1980; Wang et al., 2017). Há limitações nestes testes, pois os gatos, quando em um curso agudo da doença, ainda não desenvolveram anticorpos em nível detectável ou têm títulos baixos de anticorpos antes da morte (Cohn e Birkenheuer, 2012).

Os ensaios de PCR podem chegar a ser até 1000 vezes mais sensíveis do que a análise do esfregaço sanguíneo por microscopia de luz, sendo aplicável para confirmação de suspeita clínica na ausência ou em níveis baixos de eritroparasitemia, e para monitorar infecções em gatos que sobreviveram a uma infecção aguda (Brown et al., 2008; Wang et al., 2017).

A PCR em tempo real pode ser utilizada no prognóstico da cytauxzoonose, uma vez que observaram-se níveis mais baixos de $\mathrm{Cq}$ em gatos que sobreviveram à infecção do que em gatos que foram a óbito (Lloret et al., 2015; Cohn et al., 2011). Os ensaios da PCR utilizam como genesalvo o 18S rRNA (Birkenheuer et al., 2006) e as regiões intergênicas ITS-1 e ITS-2 Shock et al., 2012).

A PCR pode auxiliar ainda na diferenciação de patógenos que podem ter morfologia semelhantes a de Cytauxzoon spp., haja vista que patógenos do gênero Babesia spp. (Spada et al., 2014; André et al., 2014; 2015; Malheiros et al., 2016) e Theileria spp. (André et al., 2014; André et al., 2015) já foram detectados em gatos domésticos.

\section{Tratamento}

Gatos infectados por Cytauxzoon spp. têm um prognóstico grave, portanto deve ser iniciado o tratamento para todos os gatos suspeitos antes da confirmação do diagnóstico etiológico (Cohn et al., 2011; Conner et al., 2015; Sherrill et al., 2015).

A associação de atavaquona $(15 \mathrm{mg} / \mathrm{kg}$, via oral, $8-8 \mathrm{~h}$, por $10 \mathrm{dias})$ e azitromicina $(10 \mathrm{mg} / \mathrm{Kg}$, via oral, $24-24 \mathrm{~h}$, por 10 dias) mostrou bons resultados, com taxa de sobrevivência de $60 \%$. Por outro lado, a utilização de imidocarb $(3,5 \mathrm{mg} / \mathrm{Kg}$, via intramuscular, a cada 7 dias, associada com atropina na dose $0,05 \mathrm{mg} / \mathrm{Kg}$ pela via subcutânea 15 minutos antes da aplicação de imidocarb) mostrou taxa de sobrevivência de 26\% (Cohn et al., 2011).

Em pacientes com dor, é ideal que a terapia analgésica seja realizada com o uso de buprenorfina $(0,01 \mathrm{mg} / \mathrm{kg}$, via intravenosa $8-8 \mathrm{~h})$, além do uso de heparina (200 UI/kg via subcutânea, 8-8h) como profilática ou terapêutica para coagulação intravascular disseminada (Lloret et al., 2015; Sherrill et al., 2015).

A transfusão sanguínea pode ser utilizada em casos de anemia, e em casos de taquipneia e dispneia é necessária a suplementação de oxigênio (Wardrop et al., 2016; Sherrill et al., 2015).

\section{Profilaxia}

Não há nenhuma vacina comercialmente disponível até o momento, embora já tenham sido conduzidos estudos que demonstraram que os produtos dos genes $c f 30, c f 63, c f 58$ e $c f 76$ de $C$. felis podem ser os primeiros antígenos a serem utilizados em vacinas (Tarigo et al., 2013; Khana et al., 2018). Outra limitação para o desenvolvimento de vacinas é que ainda não foi obtido êxito no cultivo in vitro de $C$. felis (Schreeg et al., 2018).

A prevenção é mais focada na repelência de carrapatos nos gatos com o uso de fipronil de uso tópico, colar acaricida com associação de imidacloprida $10 \%$ associada à flumetrina $4,5 \%$ ou solução tópica de selamectina associada a sarolaner (Reichard et al., 2013; Sherrill et al., 2015; Reichard et al., 2018; Zou et al., 2019).

\section{Considerações Finais}

Antes tida como uma doença com letalidade próxima de $100 \%$, atualmente já se observa que existam gatos que podem sobreviver à infecção aguda, tendo como base os cuidados de suporte e associações de fármacos anti-protozoários. No Brasil, a despeito da detecção molecular do agente em gatos domésticos, inexistentes são os casos confirmados de cytauxzoonose nesta espécie animal. Parasitemia baixa e persistente em gatos domésticos no Brasil pode sugerir uma baixa patogenicidade da espécie de Cytauxzoon sp. na América do Sul, a despeito da proximidade filogenética de sequências parciais do gene $18 \mathrm{~S}$ rRNA de amostras brasileiras de Cytauxzoon sp. quando comparadas àquelas de isolados de $C$. felis dos EUA.

A cytauxzoonose necessita mais do que tratamentos terapêuticos, mas sim de medidas profiláticas, de modo com que seja reduzida ou 
afastada a exposição dos felídeos aos carrapatos vetores. Em regiões nas quais ainda não se identificaram os vetores, estudos posteriores deverão identificar quais os potenciais vetores.

Portanto, observa-se que os parasitas do gênero Cytauxzoon spp. vem apresentando uma expansão na sua área de ocorrência, devido principalmente ao diagnóstico por biologia molecular que tem se aperfeiçoado e se tornado acessível em diversas partes do mundo.

Uma atenção especial deve ser dada a pacientes da família Felidae que tenham sinais de apatia, febre alta, anorexia e uma evolução rápida da doença, especialmente quando se tem dados epidemiológicos favoráveis à ocorrência de exposição a artrópodes vetores. Destaca-se que quanto mais rapidamente forem estabelecidos cuidados veterinários de suportes e terapêutica, maiores as possibilidades de sobrevivência do paciente.

\section{Referências}

Allen, K.E.; Thomas, J.E.; Wohltjen, M.L; Reichard, M.V. Transmission of Cytauxzoon felis to domestic cats by Amblyomma americanum nymphs. Parasites \& Vectors, 12: 28, 2019.

Alho, A.M.; Silva, J.; Fonseca, M.J.; Santos, F.; Nunes, C.; De Carvalho, L.M.; Rodrigues, M.; Cardoso, L. First report of Cytauxzoon sp. infection in a domestic cat from Portugal. Parasites \& Vectors, 9: 220, 2016.

Alvarado-Rybak, M.; Solano-Gallego, L.; Millán, J. A review of piroplasmid infections in wild carnivores worldwide: importance for domestic animal health and wildlife conservation. Parasites \& Vectors, 9(1): 538, 2016.

André, M.R.; Adania, C.H.; Machado, R.Z.; Allegretti, S.M.; Felippe, P.A.N.; Silva, K.F.; Nakaghi, A.C.H.; Dagnone, A.S. Molecular detection of Cytauxzoon spp. in asymptomatic Brazilian wild captive felids. Journal of Wildlife Diseases, 45(1): 234-237, 2009.

André, M.R.; Denardia, N.C.B.; De Sousa, K.C.M.; Gonçalves, L.R.; Henrique, P.C.; Ontivero, C.R.G.R.; Gonzalez, I.H.L.; Nery, C.V.C.; Chagas, C.R.F.; Monticelli, C.; De Santis, C.G.A.; Machado, R.Z. Arthropodborne pathogens circulating in free-roaming domestic cats in a zoo environment in Brazil. Ticks and Tick-borne Diseases, 5(5): 545-551, 2014.
André, M.R.; Herrera, H.M.; Fernandes, S.J.; Sousa, K.C.; Gonçalves, L.R.; Domingos, I.H.; De Macedo, G.C.; Machado, R.Z. Tick-borne agents in domesticated and stray cats from the city of Campo Grande, state of Mato Grosso do Sul, midwestern Brazil. Ticks and Tick-borne Diseases, 6(6): 779-786, 2015.

André, M.R.; Filgueira, K.D.; Calchi, A.C.; Sousa, K.C.M.; Gonçalves, L.R.; Medeiros, V.B.; Ximenes, P.A.; Lelis, I.C.N.G.; Meireles, M.V.N.; Machado, R.Z. Co-infection with arthropod-borne pathogens in domestic cats. Revista Brasileira de Parasitologia Veterinária, 26(4): 525-531, 2017.

Antunes, T.R.; Da Silveira, A.W.; De Oliveira, G.G.; Rezende, A.S.; Azuaga, L.B.S.; De Souza, M.L.; De Oliveira, D.R.; Netto, C.R.M.C.; Godoy, K.C.S.; Ramos, C.A.N.; De Souza, A.I. Infecção natural por Cytauxzoon felis em onça parda (Puma concolor) de vida livre proveniente da região sudoeste de Mato Grosso do Sul, Brasil. PUBVET, 12(1): 1-5, 2018.

Aschenbroich, S.A.; Rech, R.R.; Sousa, R.S.; Carmichael, K.P.; Sakamoto, K. Pathology in practice: Cytauxzoon felis infection. Journal of the American Veterinary Medical Association, 240(2): 159-161, 2012.

Birkenheuer, A.J.; Marr, H.; Alleman, A.R.; Levy, M.G.; Breitschwerdt, E.B. Development and evaluation of a PCR assay for the detection of Cytauxzoon felis DNA in feline blood samples. Veterinary Parasitology, 137(1-2): 144-149, 2006.

Blouin, E.F.; Kocan, A.A.; Glenn, B.L.; Kocan, K.M.; Hair, J.A Transmission of Cytauxzoon felis Kier, 1979 from bobcats, Felis rufus (Schreber), to domestic cats by Dermacentor variabilis (Say). Journal of Wildlife Diseases, 20(3): 241-242, 1984.

Blouin, E.F.; Kocan, A.A.; Kocan, K.M.; Hair, J. Evidence of a limited schizogonous cycle for Cytauxzoon felis in bobcats following exposure to infected ticks. Journal of Wildlife Diseases, 23(3): 499-501, 1987.

BRASIL. Instituto Brasileiro de Geografia e Estatística. Pesquisa Nacional de Saúde: acesso e utilização dos serviços de saúde, acidentes e violências: Brasil, grandes regiões e unidades da federação. Rio de Janeiro: Instituto Brasileiro de Geografia e Estatística, 2015. Disponível em: 
<https://biblioteca.ibge.gov.br/visualizacao/livr os/liv94074.pdf>. Accesso em: 14 fev. 2018.

Brown, H.M.; Latimer, K.S.; Erikson, L.E.; Cashwell, M.E.; Britt, J.O.; Peterson, D.S. Detection of persistent Cytauxzoon felis infection by polymerase chain reaction in three asymptomatic domestic cats. The Journal of Veterinary Diagnostic Investigation, 20(4): 485-488, 2008.

Carli, E.; Trotta, M.; Chinelli, R.; Drigo, M.; Sinigoi, L.; Tosolini, P.; Furlanello, T.; Millotti, A.; Caldin, M.; Solano-Gallego, L. Cytauxzoon sp. infection in the first endemic focus described in domestic cats in Europe. Veterinary Parasitology, 183(3-4): 343-352, 2012.

Carli, E.; Trotta, M.; Bianchi, E.; Furlanello, T.; Caldin, M.; Pietrobelli, M.; Solano-Gallego, L. Cytauxzoon sp. infection in two free ranging young cats: clinicopathological findings, therapy and follow up. Türkiye Parazitoloji Dergisi, 38(3): 185-189, 2014.

Clarke, L.L.; Rissi, D.R. Neuropathology of natural Cytauxzoon felis infection in domestic cats. Veterinary Pathology, 52(6):1167-1171, 2015.

Clarke, L.L.; Krimer, P.M; Rissi, D.R. Glial changes and evidence for apoptosis in the brain of cats infected by Cytauxzoon felis. Journal of Comparative Pathology, 156(2-3): 147-151, 2017.

Conner, B.J.; Hanel, R.M.; Brooks, M.B.; Cohn, L.A.; Birkenheuer, A.J. Coagulation abnormalities in 5 cats with naturally occurring cytauxzoonosis. Journal of Veterinary Emergency and Critical Care, 25(4): 538545, 2015.

Cohn, L.A. Infecções por Cytauxzoon. In: August, J.R. Medicina interna felina. $6^{\mathrm{a}}$ ed. Rio de Janeiro: Elsevier Saunders, 2011. p. 27-35.

Cohn, L.A.; Birkenheuer, A.J.; Brunker, J.D.; Ratcliff, E.R.; Craig, A.W. Efficacy of atovaquone and azithromycin or imidocarb dipropionate in cats with acute cytauxzoonosis. Journal of Veterinary Internal Medicine, 25(1): 55-60, 2011.

Cohn, L.A.; Birkenheuer, A.J. Cytauxzoonosis. In: Greene, C.E, editor. Infectious diseases of the dog and cat. $4^{\text {th }}$ Ed. St Louis: Elsevier Saunders, 2012. p. 764-771.

Cowell, R.L.; Fox, J.C.; Panciera, R.J.; Tyler, R.D. Detection of anticytauxzoon antibodies in cats infected with a Cytauxzoon organism from bobcats. Veterinary Parasitology, 28(1-2): 4352, 1988.

Criado-Fornelio, A.; Martinez-Marcos, A.; BulingSarana, A.; Barba-Carretero, J.C. Molecular studies on Babesia, Theileria and Hepatozoon in southern Europe. Part II. Phylogenetic analysis and evolutionary history. Veterinary Parasitology, 114(3): 173-194, 2003.

Criado-Fornelio, A.; Buling, A.; Pingret, J.L.; Etievant, M.; Boucraut-Baralon, C.; Alongi, A.; Agnone, A.; Torina, A. Hemoprotozoa of domestic animals in France: prevalence and molecular characterization. Veterinary Parasitology, 159(1): 73-76, 2009.

De Sousa, K.C.M.; Fernandes, M.P.; Herrera, H.M.; Freschi, C.R.; Machado, R.Z.; André, M.R. Diversity of piroplasmids among wild and domestic mammals and ectoparasites in Pantanal wetland, Brazil. Ticks and TickBorne Diseases, 9(2): 245-253, 2018.

Díaz-Regañón, D.; Villaescusa, A.; Ayllón, T.; Rodríguez-Franco, F.; Baneth, G.; CallejaBueno, L.; García-Sancho, M.; Agulla, B.; Sainz, A. Molecular detection of Hepatozoon spp. and Cytauxzoon sp. in domestic and stray cats from Madrid, Spain. Parasites \& Vectors, 10: 112, 2017.

Driscoll, C.A.; Clutton-Brock, J.; Kitchen, A.C.; O'Brien, E.J. The taming of the domestic cat. Genetic and archaeological findings hint that wildcats became housecats earlier--and in a different place--than previously thought. Scientific American, 300 (6): 68-75, 2009.

Filoni, C.; Catão-Dias, J.L.; Cattori, V.; Willi, B.; Meli, M.L.; Corrêa, S.H.R.; Marques, M.C.; Adania, C.H.; Silva, J.C.R.; Marvulo, M.F.V.; Ferreira Neto, J.S.; Durigon, E.L.; De Carvalho, V.M.; Coutinho, S.D.; Lutz, H.; HofmannLehmann, R. Surveillance using serological and molecular methods for the detection of infectious agents in captive Brazilian neotropic and exotic felids. The Journal of Veterinary Diagnostic Investigation, 24(1): 166-173, 2012.

Furtado, M.M.; Taniwaki, S.A.; Metzger, B.; Dos Santos Paduan, K.; O'Dwyer, H.L.; De Almeida Jácomo, A.T.; Porfírio, G.E.O.; Silveira, L.; Sollmann, R.; Tôrres, N.M.; Ferreira Neto, J.S. Is the free-ranging jaguar (Panthera onca) a reservoir for Cytauxzoon felis in Brazil? Ticks and Tick-borne Diseases, 8(4): 470-476, 2017.

Gallusová, M.; Jirsová, D.; Mihalca, A.D.; Gherman, C.M.; D'Amico, G.; Qablan, M.A.; 
Modrý, D. Cytauxzoon infections in wild felids from Carpathian-Danubian-Pontic space: further evidence for a different Cytauxzoon species in European felids. Journal of Parasitology, 102(3): 377-380, 2016.

Grindem, C.B.; Tyler, R.D.; Cowell, R.L. The Bone marrow. In: Coweel, R.L.; Tyler, R.D.; Meinkoth, J.H.; De Nicola, D.B. Diagnostic citology and hematology of the dog and cat. $3^{\text {rd }}$ Ed. Saint Louis: Mosby Elsevier, 2008. p. 422-450.

Hodžić, A.; Alićb, A.; Duscher, G.G. High diversity of blood-associated parasites and bacteria in European wild cats in Bosnia and Herzegovina: A molecular study. Ticks and Tick-borne Diseases, 9(3): 589-593, 2018.

Jakob, W.; Wesemeier, H.H. A fatal infection in a bengal tiger resembling cytauxzoonosis in domestic cats. Journal of Comparative Pathology, 114(4): 439-444, 1996.

Jinnai, M.; Kawabuchi-Kurata, T.; Tsuji, M.; Nakajima, R.; Hirata, H.; Fujisawa, K.; Shiraki, H.; Asakawa, M.; Nasuno, T.; Ishihara, C. Molecular evidence of the multiple genotype infection of a wild Hokkaido brown bear (Ursus arctos yesoensis) by Babesia sp. UR1. Veterinary Parasitology, 173(1-2): 128-133, 2010.

Kelly, P.; Marabini, L.; Dutlow, K.; Zhang, J.; Loftis, A.; Wang, C. Molecular detection of tick-borne pathogens in captive wild felids, Zimbabwe. Parasites \& Vectors, 7: 514, 2014.

Ketz-Riley, C.J.; Reichard, M.V.; Van Den Bussche, R.A.; Hoover, J.P.; Meinkoth, J.; Kocan, A.A. An Intraerythrocytic Small Piroplasm In Wild-caught Pallas's Cats (Otocolobus manul) from Mongolia. Journal of Wildlife Diseases, 39(2): 424-430, 2003.

Khana, D.B.; Peterson D.S.; Stanton, J.B.; Schreeg, M.E.; Birkenheuer, A.J.; Tarigo, J.L. Genetic conservation of Cytauxzoon felis antigens and mRNA expression in the schizont life-stage. Veterinary Parasitology, 263: 49-53, 2018.

LeClaire, S.; Menard, S.; Berry, A. Molecular characterization of Babesia and Cytauxzoon species in wild South-African meerkats. Parasitology, 142(4): 543-548, 2014.

Legroux, J-P.; Halos,L.; René-Martellet, M.; Servonnet, M.; Pingret, J-L.; Bourdoiseau, G.; Baneth, G.; Chabanne, L. First clinical case report of Cytauxzoon sp. infection in a domestic cat in France. BMC Veterinary Research, 13: 81, 2017.
Lewis, K.M.; Cohn, L.A.; Birkenheuer, A.J. Lack of evidence for perinatal transmission of Cytauxzoon felis in domestic cats. Veterinary Parasitology, 188(1-2): 172-174, 2012.

Lloret, A.; Addie, D.D.; Boucraut-Baralon, C.; Egberink, H.; Frymus, T.; Gruffydd-Jones, T.; Hartmann, K.; Horzinek, M.C.; Hosie, M.J.; Lutz, H.; Marsilio, F.; Pennisi, M.G.; Radford, A.D.; Thiry, E.; Truyen, U.; Möstl, K. Cytauxzoonosis in cats: ABCD guidelines on prevention and management. Journal of Feline Medicine and Surgery, 17(7): 637-641, 2015.

Maia, L.M.P.; Cerqueira, A.M.F.; Macieira, D.B.; De Souza, A.M.; Moreira, N.S.; Da Silva, A.V.; Messick, J.B.; Ferreira, R.F.; Almosny, N.R.P. Cytauxzoon felis and 'Candidatus Mycoplasma haemominutum' coinfection in a Brazilian domestic cat (Felis catus). Revista Brasileira de Parasitologia Veterinária, 22(2): 289-291, 2013.

Malheiros, J.; Costa, M.M.; do Amaral, R.B.; de Sousa, K.C.M.; André, M.R.; Machado, R.Z.; Vieira, M.I.B. Identification of vector-borne pathogens in dogs and cats from Southern Brazil. Ticks and Tick Borne Diseases, 7(5): 893-900, 2016.

Meekins, J.; Cino-Ozuna A.G. Histologic identification of intraocular Cytauxzoon felis in three cats. Journal of Feline Medicine and Surgery Open Reports, 4(2): 1-5, 2018.

Meinkoth, J.; Kocan, A.A, Whitworth, L.; Murphy, G.; Fox, J.C.; Woods, J.P. Cats surviving natural infection with Cytauxzoon felis: 18 cases (1997-1998). Journal of Veterinay Internal Medicine, 14(5): 521-525, 2000.

Meinkoth, J.H.; Kocan, A.A. Feline cytauxzoonosis. Veterinary Clinics of North America: Small Animal, 35(1): 89-101, 2005.

Meli, M.L.; Cattori, V.; Martínez, F.; López, G.; Vargas, A.; Simón, M.A.; Zorrilla, I.; Muñoz, A.; Palomares, F.; López-Bao, J.V.; Pastor, J.;Tandon, R.; Willi, B.; Hofmann Lehmann, R.; Lutz, H. Feline leukemia virus and other pathogens as important threats to the survival of the critically endangered Iberian lynx (Lynx pardinus). PLoS One, 4(3): e4744, 2009.

Mendes-de-Almeida, F.; Labarthe, N.; Guerrero, J.; Faria, M.C.; Branco, A.S.; Pereira, C.D.; Barreira, J.D.; Pereira, M.J. Follow-up of the health conditions of an urban colony of freeroaming cats (Felis catus Linnaeus, 1758) in the city of Rio de Janeiro, Brazil. Veterinary Parasitology, 147(1-2): 9-15, 2007. 
Nagamori, Y.; Slovak, J.E.; Reichard, M.V.; Prevalence of Cytauxzoon felis infection in healthy free-roaming cats in north-central Oklahoma and central Iowa. Journal of Feline Medicine and Surgery Open Reports, 2(1): 14, 2016.

Nentwig, A.; Meli, M.L.; Schrack, J.; Reichler, I.M.; Riond, B.; Gloor, C.; Howard, J.; Hofmann-Lehmann, R.; Willi, B. First report of Cytauxzoon sp. infection in domestic cats in Switzerland: natural and transfusiontransmitted infections. Parasites \& Vectors, 11(1): 292, 2018.

Nietfeld, J.C.; Pollock, C. Fatal cytauxzoonosis in a free-ranging Bobcat (Lynx rufus). Journal of Wildlife Diseases, 38(3): 607-610, 2002.

O'Donoghue, P. Haemoprotozoa: Making biological sense of molecular phylogenies. International Journal for Parasitology: Parasites and Wildlife, 6(3): 241-256, 2017.

Ottoni, C.; Neer, W.V.; De Cupere, B.; Daligault, J.; Guimarães, S.; Peters, J.; Spassov, N.; Prendergast, M.E.; Boivin, N.; Morales-Muñiz, A.; Bălăşescu, A.; Becker, C.; Benecke, N.; Boroneant, A.; Buitenhuis, H.; Chahoud, J.; Crowther, A.; Llorente, L.; Manaseryan, N.; Monchot, H.; Onar, V.; Osypińska, M.; Putelat, O.; Morales, E.M.Q.; Studer, J.; Wierer, U.; Decorte, R.; Grange, T.; Geigl, E-M. The palaeogenetics of cat dispersal in the ancient world. Nature Ecology and Evolution. 1(7): 0139, 2017.

Peixoto, P.V.; Soares, C.O.; Scofield, A.; Santiago, C.D.; Franca, T.N.; Barros, S.S. Fatal cytauxzoonosis in captive-reared lions in Brazil. Veterinary Parasitology, 145(3-4): 383-387, 2007.

Pollard, D.A.; Reichard, M.V.; Cohn, L.A.; James, A.M.; Holman, P.J. Genetic variability of cloned Cytauxzoon felis ribosomal RNA ITS1 and ITS2 genomic regions from domestic cats with varied clinical outcomes from five states.Veterinary Parasitology, 244: 136-143, 2017.

Raghavan, R.K.; Almes, K.; Goodin, D.G.; Harrington Junior, J.A.; Stackhouse Junior, P.W. Spatially heterogeneous land cover/land use and climatic risk factors of tick-borne feline cytauxzoonosis. Vector-Borne and Zoonotic Diseases, 14(7): 486-495, 2014.

Rassouli, M.; Sabouri, S.; Goudarzi, A.; Parsa, M. Cytauxzoon felis in a stray cat in Iran.
Comparative Clinical Pathology, 24(1): 7577, 2015.

Reichard, M.V.; Van Den Bussche, R.A.; Meinkoth, J.H.; Hoover, J.P.; Kocan, A.A. A new species of Cytauxzoon from pallas' cats caught in Mongolia and comments on the systematics and taxonomy of piroplasmids. Journal of Parasitology, 91(2): 420-426, 2005.

Reichard, M.V.; Baum, K.A.; Cadenhead, S.C.; Snider, T.A. Temporal occurrence and environmental risk factors associated with cytauxzoonosis in domestic cats. Veterinary Parasitology, 152(3-4): 314-320, 2008.

Reichard, M.V.; Meinkoth, J.H.; Edwards, A.C.; Snider, T.A.; Kocan, K.M.; Blouin, E.F.; Little, S.E. Transmission of Cytauxzoon felis to a domestic cat by Amblyomma americanum. Veterinary Parasitology, 161(1-2): 110-115, 2009.

Reichard, M.V.; Edwards, A.C.; Meinkoth, J.H.; Snider, T.A.; Meinkoth, K.R.; Heinz, R.E.; Little, S.E. Confirmation of Amblyomma americanum (acari: ixodidae) as a vector for Cytauxzoon felis (piroplasmorida: theileriidae) to domestic cats. Journal of Medical Entomology, 47(5): 890-896, 2010.

Reichard, M.V.; Thomas, J.E.; Arther, R.G.; Hostetler, J.A.; Raetzel, K.L.; Meinkoth, J.H.; Little, S.E. Efficacy of an imidacloprid $10 \% /$ flumethrin $4.5 \%$ collar (Seresto ${ }^{\circledR}$, Bayer) for preventing the transmission of Cytauxzoon felis to domestic cats by Amblyomma americanum. Parasitology Research, 112 (Suplemento 1): 11-20, 2013.

Reichard, M.V.; Rugg, J.J.; Thomas, J.E.; Allen, K.E.; Barret, A.W.; Murray, J.K.; Herrin, B.H.; Beam, R.A.; King, V.L.; Vatta, A.F. Efficacy of a topical formulation of selamectin plus sarolaner against induced infestations of Amblyomma americanum on cats and prevention of Cytauxzoon felis transmission. Veterinary Parasitology, 2018. doi: 10.1016/j.vetpar.2018.10.018 (aceito com doi).

Rizzi, T.E.; Reichard, M.V.; Cohn, L.A.; Birkenheuer, A.J.; Taylor, J.D.; Meinkoth, J.H. Prevalence of Cytauxzoon felis infection in healthy cats from enzootic areas in Arkansas, Missouri, and Oklahoma. Parasites \& Vectors, 8: 13, 2015.

Schreeg, M.E.; Marr, H.S.; Tarigo, J.L.; Cohn, L.A.; Bird, D.M.; Scholl, E.H.; Levy, M.G.; Wiegmann, B.M.; Birkenheuer, A.J. Mitochondrial genome sequences and 
structures aid in the resolution of piroplasmida phylogeny. PLoS One, 11(11): e0165702, 2016.

Schreeg, M.E.; Marr, H.S.; Tarigo, J.L.; Sherrill, M.K.; Outi, H.K.; Scholl, E.H.; Bird, D.M.; Vigil, A.; Hung, C.; Nakajima, R.; Liang, L.; Trieu, A.; Doolan, D.L.; Thomas, J.E.; Levy, M.G.; Reichard, M.V.; Felgner, P.L.; Cohn, L.A.; Birkenheuer, A.J. Identification of Cytauxzoon felis antigens via protein microarray and assessment of expression library immunization against cytauxzoonosis. Clinical Proteomics, 15: 44, 2018.

Shindel, N.; Dardiri, A.H.; Ferris, D.H. An indirect fluorescent antibody test for the detection of Cytauxzoon-like organisms in experimentally infected cats. Canadian Journal of Comparative Medicine, 42(4): 460-465, 1978. Shock, B.C.; Murphy, S.M.; Patton, L.L.; Shock, P.M.; Olfenbuttel, C.; Beringer, J.; Prange, S.; Grove, D.M.; Peek, M.; Butfiloski, J.W.; Hughes, D.W.; Lockhart, J.M.; Bevins, S.N.; Van de Woude, S.; Crooks, K.R.; Nettles, V.F.; Brown, H.M.; Peterson, D.S.; Yabsley, M.J. Distribution and prevalence of Cytauxzoon felis in bobcats (Lynx rufus), the natural reservoir, and other wild felids in thirteen states. Veterinary Parasitology, 175(3-4): 325-330, 2011.

Shock, B.C.; Birkenheuer, A.J.; Patton, L.L.; Olfenbuttel, C.; Beringer, J.; Grove, D.M.; Peek, M.; Butfiloski, J.W.; Hughes, D.W.; Lockhart, J.M.; Cunningham, M.W.; Brown, H.M.; Peterson, D.S.; Yabsley, M.J. Variation in the ITS-1 and ITS-2 rRNA genomic regions of Cytauxzoon felis from bobcats and pumas in the eastern United States and comparison with sequences from domestic cats. Veterinary Parasitology, 190(1-2): 29-35, 2012.

Sherrill, M.K.; Cohn, L.A. Cytauxzoonosis: diagnosis and treatment of an emerging disease. Journal of Feline Medicine and Surgery, 17(11): 940-948, 2015.

Snider, T.A.; Confer, A.W.; Payton, M.E. Pulmonary histopathology of Cytauxzoon felis infections in the cat. Veterinary Pathology, 47(4): 698-702, 2010.

Soares, H.S.; Marcili, A.; Barbieri, A.R.M.; Minervino, A.H.H.; Moreira, T.R.; Gennari, S.M.; Labruna, M.B. Novel piroplasmid and Hepatozoon organisms infecting the wildlife of two regions of the Brazilian Amazon.
International Journal for Parasitology: Parasites and Wildlife, 6(2):115-121, 2017.

Spada, E.; Proverbio, D.; Galluzzo, P.; Perego, R.; De Giorgi, G.B.; Roggero, R.; Caracappa, S. Frequency of piroplasms Babesia microti and Cytauxzoon felis in stray cats from Northern Italy. BioMed Research International, 2014: 1-6. ID 943754, 2014.

Tarigo, J.L; Scholl, E.H.; McKBird, D.; Brown, C.C.; Cohn, L.A.; Dean, G.A.; Levy, M.G.; Doolan, D.L.; Trieu, A.; Nordone, S.K.; Felgner, P.L.; Vigil, A.; Birkenheuer, A.J. A novel candidate vaccine for cytauxzoonosis inferred from comparative apicomplexan genomics. PLoS One, 8(8): e71233, 2013.

Thomas, J.E.; Ohmes, C.M.; Payton, M.E.; Hostetler, J.A.; Reichard, M.V. Minimum transmission time of Cytauxzoon felis by Amblyomma americanum to domestic cats in relation to duration of infestation, and investigation of ingestion of infected ticks as a potential route of transmission. Journal of Feline Medicine and Surgery, 20(2): 67-72, 2018.

Veronesi, F.; Ravagnan, S.; Cerquetella, M.; Carli, E.; Olivieri, E.; Santoro, A.; Pesaro, S.; Berardi, S.; Rossi, G.; Ragni, B.; Beraldo, P.; Capelli, G. First detection of Cytauxzoon spp. infection in European wildcats (Felis silvestris silvestris) of Italy. Ticks and Tick-borne Diseases, 7(5): 853-858, 2016.

Wagner, J.E. A fatal cytauxzoonosis-like disease in cats. Journal of the American Veterinary Medical Association, 168(7): 585-588, 1976.

Wagner, J.E.; Ferris, D.H.; Kier, A.B.; Wightman, S.R.; Maring, E.; Morehouse, L.G.; Hansen, R.D. Experimentally induced cytauxzoonosislike disease in domestic cats. Veterinary Parasitology, 6(4): 305-311, 1980.

Wang, J.L.; Li, T.T.; Liu, G.H.; Zhu, X.Q.; Yao, C. Two Tales of Cytauxzoon felis Infections in Domestic Cats. Clinical Microbiology Review, 30(4): 861-885, 2017.

Wardrop, K.J.; Birkenheuer, A.; Blais, M.C.; Callan, M.B.; Kohn, B.; Lappin, M.R.; Sykes, J. Update on canine and feline blood donor screening for blood-borne pathogens. Journal of Veterinary Internal Medicine, 30(1): 1535, 2016.

Weisman, J.L.; Woldemeskel, M.; Smith, K.D.; Merrill, A.; Miller, D. Blood smear from a pregnant cat that died shortly after partial 
abortion. Veterinary Clinical Pathology, 36(2): 209-211, 2007.

Zaeemi, M.; Razmi, G.R.; Khoshnegah, J. The first detection of Cytauxzoon felis in a wild cat (Felis silvestris) in Iran.Comparative Clinical Pathology, 24(1): 181-184, 2015.

Zieman, E.A.; Jiménez, F.A.; Nielsen, C.K. Concurrent examination of bobcats and ticks reveals high prevalence of Cytauxzoon felis in Southern Illinois. Journal of Parasitology, 103(4): 343-348, 2017.
Zieman, E.A.; Nielsen, C.K.; Jiménez, F.A. Chronic Cytauxzoon felis infections in wildcaught bobcats (Lynx rufus). Veterinary Parasitology, 252(15): 67-69, 2018.

Zou, F-C.; Li, Z.; Yang, J-F.; Chang, J-Y, Liu, GH.; Lv, Y., Zhu, X-Q. Cytauxzoon felis Infection in Domestic Cats, Yunnan Province, China, 2016. Emerging Infectious Diseases, 25(2): 353-354, 2019. 